each complex

disease was

found to be

associated

with a

unique set of

Mendelian

conditions

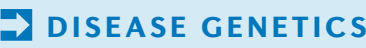

\section{A Mendelian code for complex disease}

A recent study has mined data from more than 110 million electronic medical records to give new insights into the phenotypic and genetic links between Mendelian and complex disorders, which have implications for disease-gene mapping.

Blair and colleagues analysed the phenotypic information present in large numbers of electronic medical records from the United States and

Denmark to look for co-morbidities among Mendelian and complex diseases. This analysis revealed 2,909 such associations, most of which were previously unknown, although known co-morbidities were also re-identified. Crucially, each complex disease was found to be associated with a unique set of Mendelian conditions.

The authors hypothesized that this unique 'Mendelian code' for each complex disease results from genes and pathways that are shared with the co-morbid Mendelian conditions. In support of this suggestion, analysis of results from previous genome-wide association studies (GWASs) showed that associations for complex diseases are enriched in loci that correspond to the co-morbid Mendelian conditions. This finding suggests a new way of mapping the variants that underlie complex diseases.

Another key finding from this study was an unexpectedly high number of co-morbidities between pairs of Mendelian diseases. This finding led the authors to explore genetic models that could explain the risk of complex disease in patients with more than one Mendelian phenotype. The best explanation was provided by a model in which non-additive genetic interactions in specific 'communities' of loci have crucial roles.

These findings provide new avenues for mapping complexdisease loci by following up on their Mendelian codes and have implications for understanding the genetic architectures of human diseases.

Louisa Flintoft

ORIGINAL RESEARCH PAPER Blair, D. R. et al.

A nondegenerate code of deleterious variants in mendelian loci contributes to complex disease risk. Cell 155, 70-80 (2013)

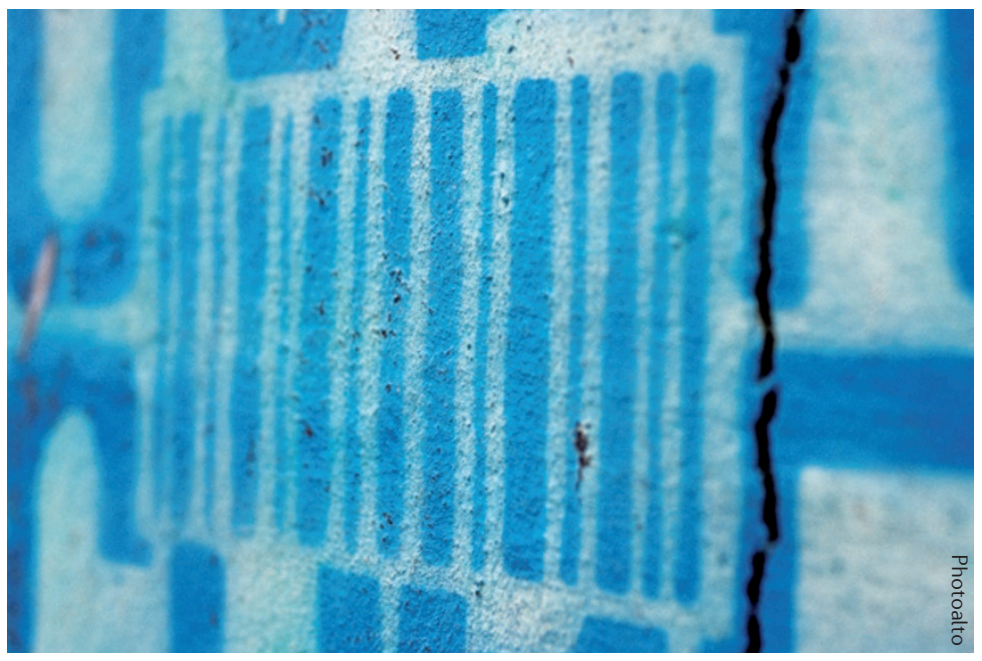

